\title{
Predictors of left main coronary artery disease in high risk patients with ACS, first episode of Non ST segment elevation MI in India
}

\author{
Sudarshan Palaparthi
}

\section{Introduction}

Ischaemic heart disease is the leading cause of death globally. ${ }^{1}$ In 2001 , ischaemic heart disease accounted for 7.1 million deaths worldwide, ${ }^{1} 5.7$ million (80\%) of which were in low-income countries. ${ }^{2,3}$ Between 1990 and 2020, these diseases are expected to increase by $120 \%$ for women and $137 \%$ for men in developing countries, compared with 30-60\% in developed countries. ${ }^{4}$ By $2010,60 \%$ of the world's heart disease is expected to occur in India. ${ }^{5}$ Furthermore, South Asians have a high prevalence of risk factors, and have ischaemic heart disease at an earlier age than do people in developed countries. ${ }^{6,7}$ Most data for patients with acute coronary syndromes are from several large registries ${ }^{8-11}$ with data on demography, treatments, and outcomes of patients in middle-income and high-income countries. There are fewer studies done in India ${ }^{12-14}$ so little is known about the demographic pattern and predictors of severe disease in ACS, particular with NSTEMI.

The natural history of significant stenosis of the left main coronary artery (LMCA) without revascularization treatment is associated with elevated mortality in patients with stable coronary disease $(50 \%$ at 3 years) ${ }^{15}$ as well as those with acute coronary syndrome (ACS) $(78.6 \%$ in patients with acute myocardial infarction $[\mathrm{AMI}]$ complicated by cardiogenic shock) ${ }^{16}$. Stenosis of the LMCA is more frequent in high-risk patients with NSTE-ACS5 $-{ }^{17}$; hence, the possibility of predicting its presence is of maximum interest. The aim of this study, performed in patients with NSTE-ACS, no known history of ischemic heart disease, and classified as at high risk, was to analyze whether any of the clinical, electrocardiographic, or biochemical parameters we use to stratify risk in NSTE-ACS at hospital admittance is related with significant LMCA stenosis in the coronary angiography undertaken later in patients of costal Andhra Pradesh, India.

\section{Material and methods}

Between may 2014 to december 2015, 423 patients with NSTE-ACS were admitted to our hospital coronary care unit in KGH vishakapatnam. Their data were analyzed and the following cases were excluded: patients with a history of ischemic heart disease, defined as those who had been hospitalized previously for myocardial infarction or unstable angina, patients treated with percutaneous or surgical coronary revascularization prior to the current event, and patients with significant valvular disease who had been diagnosed previously or during hospitalization. Patients presenting exertional angina who did not require hospital admittance with onset during the 2 months prior to the current hospitalization were, however, included. Among the total, 162 patients (38\%) had none of the above-mentioned exclusion criteria. Of these, 100 were considered at short-term high-risk following the initial clinical assessment and comprised the study group.

Patients who presented the following factors were considered at high-risk: (a) typical pain at rest that persisted despite medical treatment and involved a severe ST-segment decrease or signs of left heart failure (crepitant rales, third heart sound, or indicative features on the chest $\mathrm{x}$-ray), hypotension, severe mitral regurgitation or sustained ventricular tachycardia; (c) angina at rest of less than 24 hours of evolution and lasting for more than 30 minutes duration with ST segment decrease $\geq 2 \mathrm{~mm}$ in the anterior territory, or transient ST segment elevation; and (d) angina of less than 24 hours evolution with ST segment decrease $<2 \mathrm{~mm}$ or with a pronounced negative T-wave in the anterior territory, with positive biochemical necrosis markers (troponin I), age $>65$ years or diabetes, and at least one of the following variables on the initial work-up.

\section{Experimental Methods}

The first electrocardiogram performed in each patient following hospital admission was taken as a reference. The anterior territory was defined as the electrocardiographic tracings obtained with the V1, V2, V3, and V4, leads, the lateral territory corresponded to the I, aVL, V5, and V6 tracings and the inferior territory, to the II, III, and aVF tracings. Myocardial necrosis markers were considered pathological when the Rapid TnI test shows two coloured bands with in 15 minutes. Rapid $\mathrm{TnI}$ is designated to be positive result for $\mathrm{cTnI}$ concentration at $1.5 \mathrm{ng} / \mathrm{ml}$ or greater. The drugs commonly used for NSTE-ACS, administered during the interval between hospital admission and the coronary angiography examination, were recorded. Coronary angiography was done within the first 72 hours after hospitalization in all selected high-risk patients with 
Predictors of left main coronary artery disease in high risk patients with ACS, first episode of Non ..

NSTE-ACS, according to the aforementioned criteria. Depending on the result obtained, 2 patient groups were established: those with significant LMCA stenosis and the remaining patients. A lesion was considered to be angiographically significant when $\geq 50 \%$ of the diameter of the vessel lumen was compromised on at least 2 different views in the case of the LMCA and $\geq 70 \%$ for the remaining coronary arteries, using as a reference the adjacent segment of the vessel without angiographic lesions. ${ }^{18}$ The criteria most frequently used to establish the percentage of stenosis was the subjective assessment of any of the 3 experienced interventional radiologists who performed the coronary cardiologist.

\section{Statistical analysis:}

The observations were recorded in a proforma created for this purpose and entered in the master chart created using Microsoft Excel 2007 version. The results were presented in the form of tables and charts and expressed as proportions and percentages. Statistical Analysis was done using SPSS for windows version 21 (SPSS Inc.,Chicago, IL). Mean, median, standard deviation and Chi Squares were calculated wherever applicable. t-Test also used wherever applicable. A value of $\mathrm{P}<0.05$ was considered statistically significant.

\section{Demographic characteristics:}

\section{Results}

Among the total of 100 high-risk patients with NSTE-ACS and no known history of ischemic heart disease, 83 were men. The mean age was $55.5 \pm 8.7$ years. It is noted that $89 \%$ of LMCA disease patients are younger than 60 years, with $23 \%$ of population are constituted with age less than 50 yrs. It is noted that nearly all the patients (94\%) showed alterations on the electrocardiogram and the majority had prolonged chest pain $(63 \%)$ that appeared at rest $(77 \%)$ and was associated with elevated myocardial necrosis markers indicative of ischemia. The percentages at which the various drugs were administered in the 2 patient groups (with and without LMCA disease) are shown in Table 1.

Table 1 : Percentage of Drugs Administered in HighRisk Patients With NSTE-ACS With and Without Significant Left Main Coronary Artery Disease, During the Interval Between Hospital Admission and the Coronary Angiography Study

\begin{tabular}{|l|l|l|l|}
\hline & LMCA disease & No LMCA disease & $\mathrm{p}$ Value \\
\hline Aspirin & $100(17)$ & $100(83)$ & 1 \\
\hline Clopidogrel & $100(17)$ & $69(58)$ & $<0.0001$ \\
\hline NTG & $70(12)$ & $60(50)$ & 0.1382 \\
\hline B blokers & $23(4)$ & $55(46)$ & $<0.289$ \\
\hline Anticoagulants & $82(14)$ & $67(56)$ & 0.14954 \\
\hline Gp IIb IIIa inhibitors & $17(3)$ & $0(83)$ & $<0.001$ \\
\hline Diuretics & $47(8)$ & $12(10)$ & $<0.0001$ \\
\hline Ionotrops & $29(5)$ & $12(10)$ & 0.0029 \\
\hline ACE inhibitors & $16(14)$ & 0.0277 \\
\hline Calcium Channel blockers & $23(4)$ & $8(7)$ & 0.4693 \\
\hline
\end{tabular}

\section{Results of coronary angiography}

Seventeen patients had significant LMCA stenosis and constituted the study group. Among these patients, one had no other angiographically significant lesions, ten had additional lesions in 3 coronary arteries (left anterior descending artery [LAD], circumflex artery [CX], and right coronary artery [RCA]), three had lesions in 2 vessels ( $1 \mathrm{CX}$ and RCA and $2 \mathrm{LAD}$ and CX), and three patient had a lesion in 1 vessel (CX). The remaining patients comprised the group without LMCA disease $(n=83,83 \%)$. Among the patients with singlevessel disease $(n=31,31 \%), 19$ had a lesion in the LAD, 7 in the CX and 5 in the RCA. Among the patients with 2-vessel disease $(n=21,21 \%), 8$ had lesions in the RCA and LAD, 7 in the LAD and CX, and 6 in the RCA and LCX.
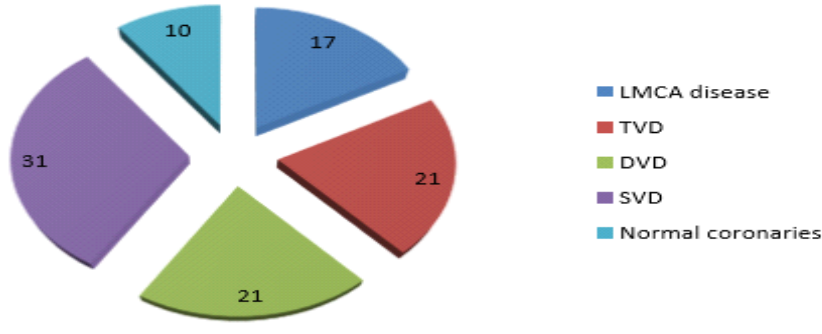
Predictors of left main coronary artery disease in high risk patients with ACS, first episode of Non ..

\section{Relationship between high risk variables and left main coronary artery disease}

In the statistical analysis, the following clinical variables used in the initial risk stratification showed a statistically significant association with the presence of LMCA: signs of obesity (central obesity ), signs of peripheral vascular disease, signs of left heart failure detected at the time of hospital admittance, cardiogenic shock at hospitalization, and lower left ventricular ejection fraction, duration of chest pain that led to hospitalization, appearance of pain while at rest. There were no significant differences between the groups with respect to the history of HTN, Smoking, DM at the time of admission or presence of ventricular arrhythmia in the first 24 hours of the ischemic event. There is a statistically significant correlation between the electrocardiographic alterations like STE in aVR $(+/-\mathrm{V} 1)$ coupled with ST depression in other leads with left main coronary artery disease .

Table 2: High-Risk Patients With NSTE-ACS and No History of Ischemic Heart Disease (n=100) Stratified According to the Criteria Stated in the Methods Section. Associations of the Various Clinical,

Electrocardiographic, and Biochemical Variables Obtained in the Initial Work-Up With the Presence of

Significant Left Main Coronary Artery Disease

\begin{tabular}{|l|l|l|l|}
\hline & LMCA disease & No LMCA disease & p value \\
\hline Men & $9(76 \%)$ & $63(75 \%)$ & 0.96 \\
\hline Age $>$ 65 & $5(29 \%)$ & $29(22 \%)$ & 0.78361 \\
\hline DM & $10(58 \%)$ & $34(41 \%)$ & 0.66433 \\
\hline PVD & $12(70 \%)$ & $21(26 \%)$ & $<0.0001$ \\
\hline HTN & $9(52 \%)$ & $61(73 \%)$ & 0.62093 \\
\hline Smoking & $8(47 \%)$ & $47(57 \%)$ & 0.67123 \\
\hline Obesity & $14(82 \%)$ & $45(54 \%)$ & $<0.0001$ \\
\hline Chest pain $>$ 30 min & $14(88 \%)$ & $49(60 \%)$ & $<0.0001$ \\
\hline Chest pain at rest & $16(94 \%)$ & $61(74 \%)$ & 0.0001 \\
\hline LV failure & $14(82 \%)$ & $13(16 \%)$ & $<0.0001$ \\
\hline Cardiogenic shock & $6(35 \%)$ & $4(5 \%)$ & $<0.0001$ \\
\hline VT & $1(6 \%)$ & $5(6 \%)$ & 1 \\
\hline EF $<55 \%$ & $11(64 \%)$ & $26(31 \%)$ & 0.001 \\
\hline ECG variables & & $53(65 \%)$ & \\
\hline ECG changes in ant leads & $11(64 \%)$ & $25(30 \%)$ & 0.8825 \\
\hline ECG changes in inf leads & $9(52 \%)$ & $27(32 \%)$ & 0.001 \\
\hline ECG changes in lateral leads & $7(41 \%)$ & $9(10 \%)$ & 0.1826 \\
\hline ST elevation in AVR & $5(30 \%)$ & & 0.0004 \\
\hline
\end{tabular}

\section{Discussion}

In this study, involving 100 high-risk patients with NSTE-ACS and no history of ischemic heart disease as defined above, $17 \%$ presented significant LMCA stenosis, In the PRISM-PLUS study, ${ }^{19}$ the proportion of patients with significant LMCA disease was $10 \%$ in the group with highest values on the TIMI scale. In contradiction to $\mathrm{CASS}^{20}$ study which has shown an association between advanced age and the presence of LMCA disease in the present study $89 \%$ of LMCA disease patients are younger than 65 years, with $23 \%$ of population are constituted with age less than $50 \mathrm{yrs}$. CAD in South Asians is usually premature, aggressive, severe, diffuse, and often follows a malignant course in this population ${ }^{21}$. Three-vessel disease is common even among premenopausal Asian Indian women ${ }^{22}$. Early development of malignant atherosclerosis results in premature myocardial infarction (MI) and death, with the first MI occurring about 7 to 10 years earlier ${ }^{21}$. The excess burden of CAD among South Asians appears to be primarily due to dyslipidemia that is characterized by high levels of apolipoprotein (apo) B, triglycerides (TG), and Lp(a); borderline high levels of low-density lipoprotein cholesterol (LDL-C); and low levels of high-density lipoprotein cholesterol (HDL-C) and apoA1. Unlike in other populations, Asian Indian vegetarians and nonvegetarians have a similar pattern of dyslipidemia and similar rates of CAD. Although there is no statistically significant correlation between diabetes mellitus and LMCA disease a sub group analysis has shown that diabetic patients are having multiple vessel disease rather than single vessel disease or no significant disease. With regard to obesity, central obesity is found in $82 \%$ of LMCA disease group where as mean BMI in that group is 26. In moderately obese individuals, regional distribution appears to be an important indicator for metabolic and cardiovascular alterations since an inconstant correlation between body mass index (BMI) and these disturbances has been found ${ }^{23}$. Many prospective studies have shown that excess fat in the upper part of the body (i.e., central or abdominal), considered by Vague ${ }^{24}$ as "android or male-type obesity," more often correlates with increased mortality and risk for disorders such as diabetes, hyperlipidemia, hypertension, and atherosclerosis of coronary, cerebral, and peripheral vessels more often than the "gynoid" (lower body or gluteo-femoral or peripheral depot) female type of fat distribution ${ }^{25}$. Visceral obesity is the event that leads to insulin resistance by causing FFA levels to increase in the liver which intern lead to insulin resistance which inturn is associated with severe coronary artery diasease. Although 
Predictors of left main coronary artery disease in high risk patients with ACS, first episode of Non ..

overall obesity delineates an important risk factor for these diseases, it is recognized that body fat distribution additionally represents an independent determinant for severe coronary artery disease.

With regard to the prognostic importance of heart failure in NSTE-ACS, the GRACE registry ${ }^{26}$ has found a significant association between signs of left heart failure at the time of hospitalization and in-hospital and out-of-hospital mortality. In addition, the prognostic value of the Killip classification ${ }^{27}$ was well established for NSTE-ACS in a recent, extensive metaanalysis in which it was an independent predictor of short- and longterm mortality, with greater prognostic value than electrocardiographic alterations or elevated myocardial necrosis markers. Thus, the search for signs of left heart failure in the initial work-up of patients with NSTEACS is important. In our series, signs of left heart failure in the initial work-up of high-risk patients presenting NSTE-ACS were predictive of LMCA disease. Nevertheless, it should be taken into account (as stated in the Limitations section) that these results must be interpreted in the light of the small size of the sample The patients analyzed herein had no known history or echocardiographic signs of having presented prior episodes of myocardial infarction.

The present study found that there is statstically significant correlation between LMCA disease and electrocardiographic alterations like STE in aVR coupled with ST depression in other leads with left main coronary artery artery disease which is similar to studies done by Yamaji et al ${ }^{16}$ and Barrabes et al ${ }^{17}$ who has shown that Lead aVR ST segment elevation with less ST segment elevation in lead V1 is an important predictor of acute LMCA obstruction. ST elevation is aVR is postulated to result from Diffuse subendocardial ischaemia, with ST depression in the lateral leads producing reciprocal change in aVR.

Lastly, it should be pointed out that the medical treatment given was similar between the 2 groups studied (with and without significant LMCA involvement), except in the use of diuretics and inotropic drugs (dopamine and dobutamine),antiplatelets (GpIIbIIIa inhibitors) which were administered more frequently in the group of patients with LMCA disease (Table 1).

\section{Conclusions}

In high-risk patients with no history of ischemic heart disease presenting with NSTE-ACS who are having LMCA disease are presenting at an younger age at the time of presentation when compared to western individuals. Central obesity as measured with waist circumference is more correlated with the presence of LMCA disease. The presence of signs of left heart disease in the initial work-up was a useful predictor of LMCA disease. In the light of these results, we believe it is worthwhile to perform emergency coronary angiography in these patients, given their poor prognosis and the possibility for improvement with therapeutic procedures such as surgical or percutaneous revascularization.

\section{Limitations of the Study}

This study has the limitations that the clinical data obtained in a single center and a small sample size. This latter aspect is a quantitative limitation, but it is also an accurate reflection of our patient population and the diagnostic and therapeutic interventions we use.

\section{References}

[1]. Lopez AD, Mathers CD, Ezzati M, Jamison DT, Murray CJ. Global and regional burden of disease and risk factors, 2001: systematic analysis of population health data. Lancet 2006; 367: 1747-57.

[2]. Yusuf S, Reddy S, Ounpuu S, Anand S. Global burden of cardiovascular diseases: part I: general considerations, the epidemiologic transition, risk factors, and impact of urbanization. Circulation 2001; 104: 2746-53.

[3]. Reddy KS. Cardiovascular disease in non-Western countries. N Engl J Med 2004; 350 (24): 2438-40.

[4]. Yusuf S, Reddy S, Ounpuu S, Anand S. Global burden of cardiovascular diseases: Part II: variations in cardiovascular disease by specifi c ethnic groups and geographic regions and prevention strategies. Circulation 2001; 104: 2855-64.

[5]. Ghaff ar A, Reddy KS, Singhi M. Burden of non-communicable diseases in South Asia. BMJ 2004; 328: 807-10.

[6]. Mohan V, Deepa R, Rani SS, Premalatha G. Prevalence of coronary artery disease and its relationship to lipids in a selected population in South India: The Chennai Urban Population Study (CUPS No. 5). J Am Coll Cardiol 2001; 38: 682-87.

[7]. Joshi P, Islam S, Pais P, et al. Risk factors for early myocardial infarction in South Asians compared with individuals in other countries. JAMA 2007; 297: 286-94.

[8]. Budaj A, Brieger D, Steg PG, et al. Global patterns of use of antithrombotic and antiplatelet therapies in patients with acute coronary syndromes: insights from the Global Registry of Acute Coronary Events (GRACE). Am Heart J 2003; 146: 999-1006.

[9]. Fox KA, Goodman SG, Klein W, et al. Management of acute coronary syndromes. Variations in practice and outcome; fi ndings from the Global Registry of Acute Coronary Events (GRACE). Eur Heart J 2002; 23: 1177-89.

[10]. Steg PG, Goldberg RJ, Gore JM, et al. Baseline characteristics, management practices, and in-hospital outcomes of patients hospitalized with acute coronary syndromes in the Global Registry of Acute Coronary Events (GRACE). Am J Cardiol 2002; 90: $358-63$.

[11]. Gibson CM. NRMI and current treatment patterns for ST-elevation myocardial infarction 5. Am Heart J 2004; 148: 29-33.

[12]. George E, Savitha D, Pais P. Pre hospital issues in acute myocardial infarction. J Assoc Physicians India 2001; 49: 320-23.

[13]. George E, Hunsberger S, Savitha D, Pais P. Treatment of acute myocardial infarction: does the type of hospital make a diff erence? PPAMI Study Group. Indian Heart J 1999; 51: 161-66.

[14]. Jose VJ, Gupta SN. Mortality and morbidity of acute ST segment elevation myocardial infarction in the current era. Indian Heart J 2004; 56: 210-14 
Predictors of left main coronary artery disease in high risk patients with ACS, first episode of Non ..

[15]. Cohen MV, Gorlin R. Main left coronary artery disease:clinical experience from 1964-1974. Circulation. 1975;52:275-85

[16]. Wong SC, Sanborn T, Sleeper LA, Webb JG, Pilchik R, Hart D, et al. Angiographic findings and clinical correlates in patients with cardiogenic shock complicating acute myocardial infarction:a report from the SHOCK Trial Registry. SHould we emergently revascularize occluded Coronaries for cardiogenic shock J Am Coll Cardiol. 2000;3Suppl A:1077-83.

[17]. Mega JL, Morrow DA, Sabatine MS, Zhao XQ, Snapinn SM, DiBattiste PM, et al. Correlation between the TIMI risk score and high-risk angiographic findings in non-ST-elevation acute coronary syndromes: observations from the Platelet Receptor Inhibition in Ischemic Syndrome Management in Patients Limited by Unstable Signs and Symptoms (PRISM-PLUS) trial. Am Heart J. 2005;149:846-50. Murray CJ, Lopez AD. Alternative projections of mortality and disability by cause 1990-2020: Global Burden of Disease Study. Lancet 1997;349:1498- 504

[18]. The Principal Investigators of CASS and Their Associates. The National Heart, Lung and Blood Institute Coronary Artery Surgery Study: historical background, design, methods, the registry, the randomised trial, clinical database. Circulation. 1981;63 Suppl 1:1-

[19]. Mega JL, Morrow DA, Sabatine MS, Zhao XQ, Snapinn SM, DiBattiste PM, et al. Correlation between the TIMI risk score and high-risk angiographic findings in non-ST-elevation acute coronary syndromes: observations from the Platelet Receptor Inhibition in Ischemic Syndrome Management in Patients Limited by Unstable Signs and Symptoms (PRISM-PLUS) trial. Am Heart J. 2005; 149:846-50.

[20]. Chaitman BR, Bourassa MG, Davis K, Rogers WJ, Tyras DH, Berger R, et al. Angiographic prevalence of high-risk coronary artery disease in patient subsets (CASS). Circulation. 1981; 64:360-7.

[21]. Enas EA, Mehta J: Malignant coronary artery disease in young Asian Indians: thoughts on pathogenesis, prevention, and treatment. Clin Cardiol 1995, 18:131-135.

[22]. Enas EA, Senthilkumar A, Juturu V, Gupta R: Coronary artery disease in women. Indian Heart J 2001, 53:282-292.

[23]. Kannel WB 1985 Lipids, diabetes and coronary heart disease: insights from the Framingham Study. Am Heart J 110:1100-1107

[24]. Vague J 1947 La differe'nciation sexuelle, facteur determinant des formes de l'obe'site'. Presse me'd 55:339-340

[25]. Donahue RP, Abbott RD, Bloom E, Reed DM, Yano K 1987 Central obesity and coronary heart disease in men. Lancet 1:821-824

[26]. Steg PG, Dabbous OH, Feldman LJ, Cohen-Solal A, Aumont MC, López-Sendón J, et al. Determinants and prognostic impact of heart failure complicating acute coronary syndromes. Observations from the global registry of acute coronary events (GRACE). Circulation. 2004;109:494-9.

[27]. Killip T III, Kimball JT. Treatment of myocardial infarction in a coronary care unit: a two year experience with 250 patients. Am J Cardiol. 1967;20:457-64.

[28]. Prediction of acute left main coronary artery obstruction by 12-lead electrocardiography. ST segment elevation in lead aVR with less ST segment elevation in lead V(1). Yamaji $\mathrm{H}$ et al J Am Coll Cardiol. 2001 Nov 1;38(5):1348-54.

[29]. Prognostic value of lead aVR in patients with a first non-ST-segment elevation acute myocardial infarction. Barrabés JA, Figueras J, Moure C, Cortadellas J, Soler-Soler J. Circulation. 2003 Aug 19;108(7):814-9. Epub 2003 Jul 28. 